\title{
Erratum to: A new species of Ophiognomonia from Northern China inhabiting the lesions of chestnut leaves infected with Diaporthe eres
}

\author{
Sai Gong ${ }^{1} \cdot$ Xiuting Zhang ${ }^{1} \cdot$ Shuxia Jiang ${ }^{1} \cdot$ Chen Chen $^{1} \cdot$ Hongbing $\mathrm{Ma}^{1} \cdot$ Yang Nie $^{1}$
}

Published online: 7 April 2017

(C) German Mycological Society and Springer-Verlag Berlin Heidelberg 2017

Erratum to: Mycol Progress (2017) 16:83-91

DOI 10.1007/s11557-016-1255-Z

The original version of this article inadvertently contained a mistake as the funder of the article was not noted.

Below is the missing information:

DOI:10.1007/s11557-016-1255-z was supported by the Shandong Provincial Natural Science Foundation, China (NO. ZR2011CM033).

The authors regret their negligence.

The online version of the original article can be found at http://dx.doi.org/ $10.1007 / \mathrm{s} 11557-016-1255-\mathrm{z}$

Shuxia Jiang

jsx6206@163.com

Shandong Province Key Laboratory of Agricultural Microbiology, Shandong Province Forestry Harmful Biology Prevention and Control Center, Department of Forest Protection, College of Plant Protection, Shandong Agricultural University, Tai'an 271018, China 